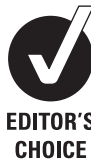

${ }^{1}$ Academic Unit of Clinical \& Experimental Sciences, University of Southampton, Southampton, UK

${ }^{2}$ NIHR Wellcome Trust Clinical Research Facility, University of Southampton and Southampton University Hospitals NHS Trust,

Southampton, UK ${ }^{3}$ Deptartment of Paediatric Infectious Diseases, Royal Alexandra Hospital for Children NHS Trust, Brighton, UK 4Pharmacy, University Hospital Southampton NHS Trust, Southampton, UK

${ }^{5}$ Medical Microbiology, University Hospital Southampton NHS Foundation Trust, Southampton, UK

\section{Correspondence to} Dr Saul N Faust, Wellcome Trust Clinical Research Facility, West Wing, Mailpoint 218, Southampton University Hospitals NHS Trust, Tremona Road, Southampton S016 6YD, UK; s.faust@soton.ac.uk

Received 22 August 2011 Accepted 13 October 2011 Published Online First 16 November 2011

\title{
Prescribing for children - taste and palatability affect adherence to antibiotics: a review
}

\author{
Dave Baguley, ${ }^{1,2}$ Emma Lim, ${ }^{2,3}$ Amanda Bevan, ${ }^{4}$ Ann Pallet, ${ }^{5}$ Saul N Faust ${ }^{1,2}$
}

\section{ABSTRACT}

The taste of an antibiotic is often not taken into account by practitioners, although there is significant evidence to show palatability correlates strongly with adherence. Many parents will be familiar with the difficulties of convincing young children to take bitter, unfamiliar medicine. Certain drugs, for example flucloxacillin, are so unpalatable that they should not be prescribed as syrups without prior 'taste testing' in an individual child, while others, such as oral cephalosporins, are accepted very well although they are more expensive with a broader antimicrobial spectrum than may be strictly necessary. Palatability is important in the broader context of global child health as regards the successful treatment of malaria, HIV and dehydration. The hidden cost of poor adherence resulting treatment failure, complications and the development of drug resistance cannot be over emphasised. Prescribing should involve parents, children and practitioners in an open discussion around the most suitable, palatable formulations for successful treatment outcomes.

\section{INTRODUCTION}

Antibiotic adherence is an important issue in the management of any paediatric infection. ${ }^{1-4} \mathrm{~A}$ number of factors usually influence the doctor's choice of antibiotic and patient adherence. These include the likely causative organism and its sensitivities, and the site and severity of infection. The child's age and known allergies, and drug route, formulation, degree of penetration, side effects and toxicology are also important issues. However, the taste of an antibiotic and the child's ability to tolerate oral drugs, although widely cited, is often not considered. Acknowledging the importance of palatability to children and parents in patient-centred management will improve adherence and influence clinical outcome.

Bitter taste is sensed by receptors that have evolved through the need to recognise a vast number of potentially harmful and structurally different compounds. ${ }^{5}$ When placed in this evolutionary context, it is not surprising that parents often find it difficult to persuade children to take bitter medicines. Commonly prescribed oral antibiotics for children include penicillins, cephalosporins and macrolides. Cephalosporins are often regarded as pleasant tasting, ${ }^{1}{ }^{6-9}$ while other penicillin-based antibiotics have a less favourable taste and aftertaste. ${ }^{710}$ The majority of doctors have not tasted every antibiotic they prescribe. We review the clinical evidence around palatability with the aim of informing routine medical practice and influencing future antimicrobial guideline development.

\section{ADHERENCE AND TASTE}

Palatability is so significant in ensuring successful administration of a course of treatment that a recent call has been made for the evaluation of palatability and taste before European marketing authorisation is granted..$^{11}$ Current European Parliament legislation ${ }^{12}$ also emphasises the importance of making new drugs more acceptable. However, palatability comes at a price as the taste of traditionally unpalatable drugs has commonly been disguised with sugar. ${ }^{13}$ Regulatory agencies and professional organisations in Europe and the USA have recommended avoidance of sugar in paediatric medicines and many artificial sweeteners have been substituted, but sweeteners themselves may also have associated adverse effects. $^{13}$

Throughout the literature, the taste, texture and aftertaste of antibiotics are cited as important considerations for children taking medicines. ${ }^{1} 2$ A questionnaire study in Japan ${ }^{14}$ of 192 families revealed non-adherence to a full course of antibiotics in approximately one-quarter of patients. The child's refusal to take the drug was the second most common reason for non-adherence after parental judgement that the child was better. Another questionnaire-based study ${ }^{15}$ of 414 primary care patients (65.9\% children) in Saudi Arabia examined non-adherence to short-term antibiotic therapy, and the reasons most frequently mentioned were the rapid improvement of symptoms, the bitter taste of the drug, forgetfulness and frequent dosing.

Better tasting antibiotics should therefore correlate with higher levels of adherence. A large study ${ }^{16}$ $(n=546)$ in Israeli paediatric outpatients assessed acceptability and adherence to the most commonly prescribed oral antibiotic regimes. Parents were interviewed by phone 10-14 days after commencing treatment. Four drugs were investigated: $11 \%$ of patients resented or refused cefaclor, $16 \%$ amoxicillin, $26 \%$ trimethoprim/sulfamethoxazole and $56 \%$ cefuroxime axetil. Unsurprisingly, this showed a positive correlation with the numbers of children completing the entire course: $85 \%$ (cefaclor), $77 \%$ (amoxicillin), 73\% (trimethoprim/sulfamethoxazole) and $67 \%$ (cefuroxime axetil).

\section{PALATABILITY}

Five different components of taste are detected by the tongue, with specific cell types possessing unique receptors capable of individually sensing bitter, salty, sour, sweet and umami tastes. ${ }^{17}$ These components excite specific neural pathways and are processed along with other immediate olfactory, visual and somatosensory inputs, 
as well as those from memory. ${ }^{5}$ Taste is therefore influenced to some degree by prior experience, and the components of taste have evolved through necessity. For example, the ability to taste sweet food allows the identification of energy-rich foodstuffs, whereas recognising a bitter taste helps detect substances unsuitable for consumption and therefore protects against naturally occurring, potentially lethal substances. Bitter taste receptors have had to evolve to recognise a vast number of structurally different compounds all capable of triggering the sensation of bitterness. ${ }^{5}$

\section{Measurement of palatability}

A variety of methods have been used in order to assess taste perception in children and subsequently draw conclusions concerning drug palatability. However, a European Medicines Evaluation Agency Report ${ }^{18}$ acknowledges the difficulty of assessing taste in children below 5 years of age. Methods to measure palatability range from the simple to the sophisticated.

The time required for a medicine to be given ${ }^{19}$ and children's own spontaneous verbal judgements after consumption ${ }^{20}$ have been considered useful and effective ways to compare responses to different flavours. Patient and parental questionnaires have been used to evaluate taste $e^{15} 16$ and to assess the acceptability $^{16}$ of antibiotic preparations. However, there are clear limitations with such methods in very young children or those unable to articulate appropriately.

The use of universal, standardised visual analog scales to quantify a child's response to the taste of a medicine (eg, the facial hedonic method) are widely described throughout the literature. 68101121 One study has shown that the hedonic method is worse than own spontaneous verbal judgements at discriminating between taste differences in children under 6 years of age, although both methods are equally effective for older children. ${ }^{21}$ Despite this, the study concluded that the hedonic scale was still preferable across all ages due to standardisation

More sophisticated methods of taste evaluation have been trialled including the ASTREE Electronic Tongue; and taste sensor systems ${ }^{22}$ which measure the electronic potential of drug solutions and have been shown to distinguish between different antibiotics.

Randomised control trials are the gold standard in comparing treatment outcomes $8911192023-25$ and have been shown to be an effective method of demonstrating antibiotic superiority in terms of both treatment outcomes and taste.

\section{Palatability of specific drugs}

A large number of studies have focused on palatability as an outcome. Certain antibiotics are widely acknowledged to have an unpleasant taste. A Japanese study ${ }^{22}$ in adults used human gustatory sensation tests to gauge the bitterness intensity of 18 common paediatric antibiotic and antiviral drugs with quinine as an unpleasant standard. Seven drugs (azithromycin, clarithromycin, erythromycin, norfloxacin, sulfamethoxazole/ trimethoprim and amantadine) were judged more bitter than quinine. Erythromycin has an unpleasant taste ${ }^{22} 26-28$ and is associated with nausea. Clarithromycin is characteristically bitter $^{28-30}$ and has faired poorly as regards palatability when directly compared ${ }^{22} 27$ to other antibiotics.

A number of studies ${ }^{16-9} 27$ including a blind taste comparison in adults of 14 paediatric antibiotic formulations and a double-blind taste comparison of 22 paediatric antibiotics, ${ }^{27}$ have found cephalosporins to be better tasting although more expensive than penicillins and macrolides. Dicloxacillin, oxacillin, erythromycin/sulfisoxazole and cefpodoxime were judged so unpleasant that they ran the risk of endangering adherence. ${ }^{27}$ Another blinded taste study cumulatively scored 12 antimicrobial suspensions; loracarbef scored highest but not significantly higher than the cephalosporins: cephalexin, cefixime and cefaclor. Again, the lowest scoring antibiotic was dicloxacillin. ${ }^{7}$ A small trial $(n=20)$ of four anti-staphylococcal antibiotics found that children's taste perception of cloxacillin (similar to fluxloxacillin in UK) was so poor as to recommend not prescribing the drug at all in the interests of adherence. ${ }^{10}$

Although data in the literature are incomplete, ${ }^{11}$ branded antimicrobial preparations often appear better tolerated than their cheaper generic equivalents.

\section{COMIMON PAEDIATRIC DISEASES}

The unpleasant taste of antibiotics is consistently cited as an obstacle to adherence and should therefore be a key consideration in the management of all common paediatric infectious conditions. ${ }^{2} 31$ However, unpleasant tasting antibiotics are recommended as first line choices in a number of common paediatric infectious diseases (see the case study described in box 1).

Penicillin V is recommended as a first line antibiotic treatment for acute streptococcal pharyngitis, although cefdinir and azithromycin have been shown to be more palatable alternatives. Azithromycin was also shown to be a more palatable alternative drug for the treatment of upper respiratory tract infections in a study of 769 children using blinded

\section{Box 1 Case study: recommendations for the} treatment of eczema

Key issue highlighted by the 2007 NICE treatment guideline: "Atopic eczema in children: management of atopic eczema in children from birth up to the age of 12 years" 39

- The NICE recommendation for first line infected eczema systemic treatment is flucloxacillin for Staphylococcus aureus and streptococcal skin infections, with erythromycin as second line treatment for children allergic to penicillin or with flucloxacillin resistance, and clarithromycin as an alternative to erythromycin with fewer side effects.

- Problems

- Oral flucloxacillin has long been recognised as being a poor taste choice, and is acknowledged by NICE as often considered unpalatable by children.

- Erythromycin has an unpleasant taste and is associated with nausea, however NICE recommend its use due to its equal efficacy with, but cheaper cost than, cephalosporins.

- Anecdotally, paediatricians may think that clarithromycin tastes better than erythromycin, however clarithromycin is characteristically bitter and has faired poorly regarding palatability when directly compared to other antibiotics.

- Clindamycin is also well known for poor palatability and adherence.

- Conclusions

- Cost plays an important part in the development of any national guideline.

- However, the hidden burden of poor adherence, subsequent re-treatment, the complications of partially treated disease and burgeoning resistance are also important considerations. 
Box 2 Acceptability and treatment dose frequency of commonly prescribed oral antibiotic agents (unpublished author experience)

- Antibiotics children will normally swallow

- Co-amoxiclav ( $\times 3 /$ day) or Augmentin Duo ( $\times 2 /$ day)

- Cefaclor, cefalexin, Amoxil (branded) (all $\times 3 /$ day)

- Co-trimoxazole

- Antibiotics children might swallow

- Penicillin V $(\times 4 /$ day $)$

- Amoxicillin (generic) $(\times 3 /$ day)

- Clarythromycin ( $\times 2 /$ day), azithromycin ( $\times 1 /$ day)

- Antibiotics children often spit out or grimace when taking

- Erythromycin $(\times 4 /$ day $)$

- Trimethoprim $(\times 2 /$ day $)$

- Rarely tolerated with good adherence

- Flucloxacillin $(\times 4 /$ day $)$

- Clindamycin ( $\times 4 /$ day)

taste tests. Although current UK primary care $^{32}$ guidelines discourage antibiotics for the treatment of acute otitis media, they acknowledge they are at times necessary. A number of randomised controlled trials have shown azithromycin and cefdinir $^{25}$ to be more palatable alternatives to amoxicillin/ clavulanate while also being easier for parents to administer. Cefdinir additionally exhibits better treatment adherence (although unavailable in the UK). Both drugs are easier for parents to administer, particularly standard azithromycin therapy which is given once daily for a short 3-day course. Amoxicillin has not been subjected to similar trials, but taste might generally be considered equivalent to amoxicillin/clavulanate formulations (box 2).

The potential benefit of using cephalosporins to enhance adherence needs to be weighed against using a less effective agent that incurs the risk of developing resistant organisms such as methicillin resistant Staphylococcus aureus (MRSA) or extended spectrum beta lactamases (ESBLs), or of Clostridium difficile infection. However, the risk of $C$ difficile and MRSA infection is much lower in children than adults and so the use of a more palatable but broader spectrum antibiotic in these circumstance is almost certainly preferable. For a child receiving multiple courses of antibiotics or requiring chemoprophylaxis with regular antimicrobials, the decision to use a broader spectrum agent should be considered individually.

\section{IMPROVING DRUG ADMINISTRATION}

The palatability of certain drugs such as flucloxacillin is so poor that we recommend that the drug not be prescribed at all in suspension form unless the child is observed swallowing a dose prior to full prescription ('taste test') and the parents can give 6 hourly medication. Models in HIV management have shown that pill swallowing training can be very successful, with $91 \%$ of $6-11$-year-old children able to swallow tablets. ${ }^{33}$ Improving children's ability to take pills has been shown to improve medication adherence, and is a useful alternative method of delivery for unpalatable drugs ${ }^{3}$ where alternatives are not available (box 3).

\section{IMPROVING TASTE}

A number of methods to improve the taste of antibiotics have been investigated. Although erythromycin A is usually
Box 3 How to (A) perform a 'taste test' and (B) explain 'pill school' to parents ${ }^{40}$

A1) Taste test in hospital: prior to switch from intravenous formulation, change to unpalatable oral formulation and observe the parent giving all doses of medication before discharge. For all beta-lactam antibiotics and macrolides, explain to the parent that they can give a repeat dose if partially spat out or vomited.

A2) Taste test in community (requires drug availability in the primary care setting): ask the practice nurse to observe the dose being given prior to issuing a prescription for an unpalatable formulation.

B) 'Pill school': Explain to the parents the use of widely available confectionary for helping the child practice swallowing tablets with water or dilute squash from small (tic tacs), through medium (small then standard Smarties) to large (chocolate or yoghourt coated raisins) size.

It is important to

i) not force the issue and ensure fun

ii) practice a little each day, do not worry if takes weeks or months

iii) if possible offer telephone back-up to the parent via a clinical nurse specialist.

manufactured as a taste-free prodrug in the form 2'-ethyl succinate, the drug is unstable and undergoes hydrolysis resulting in its unpleasant bitter tasting form. A different version of the drug, erythromycin B enol, has been proposed which has improved stability and therefore better taste. ${ }^{26}$ Microencapsulation has been studied as a way of masking the bitter taste of clarithromycin and erythromycin. ${ }^{28}$ Similarly, the production of microspheres containing azithromycin has shown promise as an effective method to hide its taste. ${ }^{34}$ Other proposed methods include the use of flavoured jellies to disguise the bitter taste of macrolides. ${ }^{30}$

\section{FURTHER CONSIDERATIONS}

The palatability of paediatric drug formulations should also be considered in the broader context, as low-resource settings carry much of the burden of paediatric infectious diseases. Oral re-hydration supplements, an important therapy in the treatment of diarrhoea, have been shown to vary significantly in palatability and acceptability to children. ${ }^{35}$

The unpleasant taste of many anti-retroviral drugs is well recognised and it remains that only a minority of anti-retroviral drugs have paediatric formulations. Palatability was shown ${ }^{4}$ to be a common reason for non-adherence to treatment in children with HIV in South India. The huge impact of non-adherence and emerging viral resistance has serious implications for the health of HIV infected children and the success of national treatment programmes. As new drugs are developed, the need for more paediatric formulations with improved palatability is paramount.

However, the majority of palatability studies are not conducted in low-resource settings and taste preferences vary significantly across cultures. ${ }^{36}$ Nonetheless, the importance of adherence in effective management remains the same. The need for local studies and the development of pleasant paediatric formulations could potentially have a substantial effect on global child morbidity. 


\section{CONCLUSION}

In summary:

- Doctors need to be more aware of the importance of taste in paediatric prescribing.

- Palatability correlates strongly with adherence.

$\triangleright$ Cephalosporins, despite their relative cost, are accepted very well.

$\triangleright$ Flucloxacillin suspension is so unpalatable that it is recommended that it is only prescribed in capsule form unless the child is observed swallowing a dose prior to full prescription ('taste test') and the parents can give 6 hourly medication.

- Children from the age of 6 can swallow pills with training (which can be carried out by parents following a short medical or nursing explanation).

- Children and families need to be involved in choosing treatments for successful outcomes.

- Prescribers and families must be aware that generic medicines may differ between prescriptions with considerable taste variability.

In 3- and 4-year-old children, familiarity and sweetness influence are the major factors in food preferences. ${ }^{37}$ The majority of children's prescribed medicines are unfamiliar and bitter, so it is not surprising that completing courses of antibiotics is difficult for both parents and children. The unpleasant taste of an antibiotic has been widely cited as a common reason for non-adherence and better tasting antibiotics correlate with higher levels of treatment completion. ${ }^{16}$ Although the evidence is primarily from the treatment of tuberculosis and HIV, ${ }^{38}$ poor antimicrobial adherence can result in treatment failure and subsequent complications, outcomes costly for both the patient and the health services. Additionally, there is a hypothetical risk of increased antibiotic resistance where a medication has not been administered effectively because of taste or dose frequency. Such is the effect of an antibiotic's taste on adherence that recent calls have been made for the evaluation of palatability and taste before European marketing approval is granted.

Doctors prescribing antibiotics for children need a higher level of awareness of the relative palatability of drugs if they have not personally tasted them. Cephalosporins, despite their relative cost, are accepted very well and therefore adherence and successful treatment are more likely.

An essential component of paediatric drug adherence is family involvement in the choice of treatment. A patient-centred approach involves effective communication and partnership between the child, parents and professionals. Open discussions around issues such as taste, formulation and dosing schedule can influence the selection of an appropriate antibiotic and the success of the treatment.

Funding This work was carried out by DB while funded by the National Institute of Health Research as an Academic Foundation Doctor at the University of Southampton, and was supported by the NIHR Wellcome Trust Clinical Research Facility.

Competing interests None.

Provenance and peer review Not commissioned; externally peer reviewed.

\section{REFERENCES}

1. Ramgoolam A, Steele R. Formulations of antibiotics for children in primary care: effects on compliance and efficacy. Paediatr Drugs 2002;4:323-33.

2. Pichichero ME. Empiric antibiotic selection criteria for respiratory infections in pediatric practice. Pediatr Infect Dis J 1997;16:S60-4.

3. Garvie PA, Lensing S, Rai SN. Efficacy of a pill-swallowing training intervention to improve antiretroviral medication adherence in pediatric patients with HIV/ AIDS. Pediatrics 2007;119:e893-9.

4. Paranthaman K, Kumarasamy N, Bella D, et al. Factors influencing adherence to anti-retroviral treatment in children with human immunodeficiency virus in South India-a qualitative study. AIDS Care 2009;21:1025-31.
5. Behrens M, Meyerhof W. Bitter taste receptors and human bitter taste perception. Cell Mol Life Sci 2006;63:1501-9.

6. Angelilli ML, Toscani M, Matsui DM, et al. Palatability of oral antibiotics among children in an urban primary care center. Arch Pediatr Adolesc Med 2000;154:267-70.

7. Demers DM, Chan DS, Bass JW. Antimicrobial drug suspensions: a blinded comparison of taste of twelve common pediatric drugs including cefixime, cefpodoxime, cefprozil and loracarbef. Pediatr Infect Dis J 1994;13:87-9.

8. Schwartz R. Enhancing children's satisfaction with antibiotic therapy: a taste study of several antibiotic suspensions. Curr Ther Res 2000; 61(8):570-81.

9. Holas C, Chiu YL, Notario G, et al. A pooled analysis of seven randomized crossover studies of the palatability of cefdinir oral suspension versus amoxicillin/ clavulanate potassium, cefprozil, azithromycin, and amoxicillin in children aged 4 to 8 years. Clin Ther 2005;27:1950-60.

10. Matsui D, Barron A, Rieder MJ. Assessment of the palatability of antistaphylococcal antibiotics in pediatric volunteers. Ann Pharmacother 1996;30:586-8.

11. Cohen R, de La Rocque F, Lécuyer A, et al. Study of the acceptability of antibiotic syrups, suspensions, and oral solutions prescribed to pediatric outpatients. Eur $J$ Pediatr 2009:168:851-7.

12. Union EPaotCotE. Regulation (EC) No 1901/2006 of the European Parliament and of the Council of the European Union of 12 December 2006 on medicinal products for paediatric use and amending Regulation (EEC) No 1768/92, Directive 2001/20/EC, Directive 2001/83/EC and Regulation (EC) No 726/2004: Chapter 3: Section 1: Article 15. Official Journal of the European Union. 2006:378/8

13. Scadding G. Pediatric allergy medications: review of currently available formulations. Curr Med Res Opin 2009;25:2069-79.

14. Sunakawa K, Akita $\mathrm{H}$, Iwata S, et al. Rational use of oral antibiotics for pediatric infections. Infection 1995;23 Suppl 2:S74-8.

15. al-Shammari SA, Khoja T, al-Yamani MJ. Compliance with short-term antibiotic therapy among patients attending primary health centres in Riyadh, Saudi Arabia. $J$ R Soc Health 1995;115:231-4.

16. Dagan $\mathbf{R}$, Shvartzman P, Liss Z. Variation in acceptance of common oral antibiotic suspensions. Pediatr Infect Dis J 1994;13:686-90.

17. Chandrashekar J, Hoon MA, Ryba NJ, et al. The receptors and cells for mammalian taste. Nature 2006;444:288-94.

18. CHMP. Reflection paper: Formulations of choice for the paediatric population, 2006. Available from: http://www.emea.europa.eu/pdfs/human/ paediatrics/19481005en.pdf.

19. Uhari M, Eskelinen L, Jokisalo J. Acceptance of antibiotic mixtures by infants and children. Eur J Clin Pharmacol 1986;30:503-4.

20. Bagger-Sjöbäck D, Bondesson G. Taste evaluation and compliance of two paediatric formulations of phenoxymethylpenicillin in children. Scand J Prim Health Care 1989;7:87-92.

21. Sjövall J, Fogh A, Huitfeldt B, et al. Methods for evaluating the taste of paediatric formulations in children: a comparison between the facial hedonic method and the patients' own spontaneous verbal judgement. Eur J Pediatr 1984:141:243-7.

22. Ishizaka T, Miyanaga Y, Mukai J, et al. Bitterness evaluation of medicines for pediatric use by a taste sensor. Chem Pharm Bull 2004;52:943-8.

23. El-Chaar GM, Mardy G, Wehlou K, et al. Randomized, double blind comparison of brand and generic antibiotic suspensions: II. A study of taste and compliance in children. Pediatr Infect Dis J 1996;15:18-22.

24. Pichichero M, Aronovitz GH, Gooch WM, et al. Comparison of cefuroxime axetil, cefaclor, and amoxicillin-clavulanate potassium suspensions in acute otitis media in infants and children. South Med J 1990;83:1174-7.

25. Block SL, Schmier JK, Notario GF, et al. Efficacy, tolerability, and parent reported outcomes for cefdinir vs. high-dose amoxicillin/clavulanate oral suspension for acute otitis media in young children. Curr Med Res Opin 2006;22:1839-47.

26. Bhadra PK, Morris GA, Barber J. Design, synthesis, and evaluation of stable and taste-free erythromycin proprodrugs. J Med Chem 2005;48:3878-84.

27. Steele RW, Estrada B, Begue RE, et al. A double-blind taste comparison of pediatric antibiotic suspensions. Clin Pediatr (Phila) 1997;36:193-9.

28. Zgoulli S, Grek V, Barre G, et al. Microencapsulation of erythromycin and clarithromycin using a spray-drying technique. J Microencapsul 1999;16:565-71.

29. Tanigake A, Miyanaga Y, Nakamura T, et al. The bitterness intensity of clarithromycin evaluated by a taste sensor. Chem Pharm Bull 2003;51:1241-5.

30. Tsuji E, Uchida T, Fukui A, et al. Evaluation of bitterness suppression of macrolide dry syrups by jellies. Chem Pharm Bull 2006;54:310-4.

31. Brook I. Current issues in the management of acute bacterial sinusitis in children Int J Pediatr Otorhinolaryngol 2007;71:1653-61.

32. NICE. CG69: Respiratory tract infections - antibiotic prescribing: Prescribing of antibiotics for self-limiting respiratory tract infections in adults and children in primary care: 2.2.3 The clinical effectiveness and cost effectiveness of antibiotic management strategies for RTIs: Recommendation number 1.1.32008. Available from: http://www.nice.org.uk/nicemedia/pdf/CG69FullGuideline.pdf. 
33. Meltzer E0, Welch MJ, Ostrom NK. Pill swallowing ability and training in children 6 to 11 years of age. Clin Pediatr (Phila) 2006;45:725-33.

34. Hu L, Pan J, Liu C, et al. Preparation, characterization and taste-masking properties of microspheres containing azithromycin. J Pharm Pharmacol 2009:61:1631-5

35. Freedman SB, Cho D, Boutis K, et al. Assessing the palatability of oral rehydration solutions in school-aged children: a randomized crossover trial. Arch Pediatr Adolesc Med 2010;164:696-702.

36. Craig SR, Adams LV, Spielberg SP, et al. Pediatric therapeutics and medicine administration in resource-poor settings: a review of barriers and an agenda for interdisciplinary approaches to improving outcomes. Soc Sci Med 2009;69:1681-90.
37. Birch LL. Development of food acceptance patterns in the first years of life. Proc Nutr Soc 1998;57:617-24.

38. Castro H, Judd A, Gibb DM, et al.; Pursuing Later Treatment Options II (PLATO II) project team for the Collaboration of Observational HIV Epidemiological Research Europe (COHERE). Risk of triple-class virological failure in children with HIV: a retrospective cohort study. Lancet 2011;377:1580-7.

39. NICE. CG57: Atopic eczema in children: Management of atopic eczema in children from birth up to the age of 12 years, 2007. Available from: http://www.nice.org. uk/Guidance/CG57.

40. Children's HIV Association. Pill swallowing technique. http://chiva.org.uk/ professionals/health/guidelines/adherence/pill-factsheet.html (accessed 08 Aug 2011). 